TRANS · NÚM. II $\cdot 2007$

ARTICULOS · 197-214
La propuesta metodológica para la revisión de traducciones que presentamos en este artículo se caracteriza por integrar, además de los parámetros de revisión, la modalidad de revisión que se va a realizar, en función de los aspectos genéricos del texto de llegada que se pretendan verificar, y el perfil del revisor más apropiado para llevar a cabo esta tarea. Nuestra propuesta es la conclusión, por una parte, de un análisis descriptivo, comparativo y crítico sobre la revisión de traducciones en la Traductología y, por otra, de la valoración global de los resultados del estudio de tres casos. Para una mejor comprensión de esta propuesta, expondremos los principios generales de la revisión en los que nos hemos basado para determinar, a continuación, los parámetros que consideramos de mayor utilidad para revisar una traducción.

PALABRAS CLAVE: revisión de traducciones, principios para la revisión, parámetros de revisión, modalidades de revisión y grados de revisión.

\title{
Propuesta metodológica para la revisión de traducciones: principios generales y parámetros
}

Silvia Parra Galiano

Universidad de Granada
In this article we present a methodological proposal for translation revision which integrates, both the revision parameters and revision methods to be used, according to the generic aspects of the target text to be revised, and the most appropriate profile for the reviser in order to fulfil this task. Our integrated methodological proposal for trans/ation revision is the result of 1) a descriptive, comparative and critical analysis of translation revision in Translation Studies and 2) the assessment of the overall results of three case studies. We will also put forward an explanation concerning the general revision principles we have used as a basis for establishing the most useful parameters for translation revision.

KEY WORDS: translation revision, revision principles, revision parameters, revision degrees and revision methods. 


\section{INTRODUCCIÓN}

La propuesta metodológica integrada para la revisión de traducciones que presentamos está basada, por una parte, en un análisis descriptivo, comparativo y crítico sobre la revisión de traducciones en la Traductología y, por otra, en la valoración global de los resultados del estudio de tres casos, relacionados con la práctica de la revisión en el ámbito profesional.

Los casos de estudio fueron los siguientes: Caso r: La evaluación de la calidad y la revisión de las traducciones externas en el Centro de Traducción de los Órganos de la Unión Europea; Caso 2: La revisión en la norma alemana DIN 2345 y Caso 3: Criterios de revisión en la norma LISA QA Model para el Aseguramiento de la calidad en el sector de la localización y aplicación a la traducción.

En la denominación de nuestra propuesta metodológica para la revisión de traducciones figura el adjetivo «integrada» por dos motivos:

a) El primero, porque contiene la mayoría de los principios generales y los parámetros de revisión que hemos analizado, tanto en las escasas obras monográficas sobre el tema como en el estudio de los tres casos referidos, junto a los aspectos más interesantes de las reflexiones y estudios de diversos autores y nuestra propia aportación

b) El segundo, porque se caracteriza por integrar, además de los parámetros de revisión, la modalidad de revisión que se va a realizar, en función de los aspectos genéricos del texto de llegada ( $\mathrm{TL}$ ) que se pretendan verificar, y el correspondiente perfil del revisor.

Emulando algunas de las interesantes reflexiones que hace Darbelnet (I980) en un artículo sobre la teoría y práctica de la traducción profesional, iniciaremos este artículo con varias consideraciones.
Por una parte, el diseño y la elaboración de esta propuesta nos han obligado a utilizar una terminología que hemos de considerar provisional, hasta que no cuente con un cierto consenso, habida cuenta de la práctica inexistencia de literatura específica en español sobre la revisión de traducciones.

Por otra, no pretendemos mostrar una teoría yuxtapuesta a las analizadas. Bien al contrario, nuestra propuesta es integradora puesto que constituye el resultado del análisis de las teorías y propuestas formuladas, fundamentalmente, por Horguelin (1985), Horguelin y Brunette (r998), Hosington y Horguelin (I980) y Mossop (200ra), de nuestra propia reflexión y, finalmente, de nuestra experiencia en el ámbito profesional y académico de la traducción.

Por este motivo, preferimos mostrar una serie de principios ya que, contrariamente a lo que ocurre con las teorías, de acuerdo con Darbelnet (I980: 394), el uso de los principios resulta más práctico puesto que se pueden aislar, concretizar, asumir o rechazar por separado.

Finalmente, hemos de considerar que la revisión de traducciones ha adquirido una nueva dimensión al institucionalizarse debido a que se ha constituido como una especialización en la profesión de traductor. Recordemos que, entre otros factores, el incremento del número de traductores con una experiencia limitada, tanto en organismos oficiales como en la empresa privada, ha impuesto el control de la calidad del trabajo que realizan como algo necesario y generalizado. De ahí que, desde el punto de vista teórico, la revisión de traducciones se muestre como un elemento crucial para reflexionar sobre las condiciones en las que se desarrolla el proceso de traducción y sobre la justificación y manera de realizar ese control de la calidad generalizado. Para justificar esta idea, 
a continuación, mencionaremos los objetivos que nos propusimos para el estudio de los tres casos y expondremos brevemente los resultados globales más relevantes para la elaboración de nuestra propuesta.

\section{RESULTADOS GLOBALES DEL ESTUDIO DE TRES CASOS}

Los objetivos comunes para el estudio de los tres casos consistieron en conocer:

I) La relevancia que se otorga a la revisión de traducciones en el marco del uso de sistemas de calidad en la prestación de servicios de traducción

2) La relación que existe entre la revisión de traducciones y otros procedimientos para valorar la calidad de la traducción en el marco de los sistemas de calidad estudiados

3) En qué momento y por qué motivo se recurre a la revisión en el marco de los sistemas de calidad estudiados, considerando que, en dichos sistemas, se utilizan diversos procedimientos para valorar y garantizar la calidad de la traducción y

4) Qué parámetros se utilizan para revisar las traducciones en los sistemas de calidad analizados.

Una vez analizados los casos, hemos concluido que los tres sistemas de calidad estudiados: el sistema implantado en el Centro de traducción de los Órganos de la Unión Europea (ст), la norma alemana DIN 2345 y la norma LISA QA Model para el Aseguramiento de la Calidad en el sector de la localización, consideran la revisión de la traducción, como un procedimiento inherente a dichos sistemas de calidad. Sin embargo, la importancia de la revisión se materializa y difiere en los tres sistemas estudiados en dos sentidos:

I) En cuanto a la obligación de realizarla: es obligatoria, siempre, en las normas DIN 2345 y
LISA QA Model ${ }^{\mathrm{I}}$ y, sólo, en ciertos casos en el CT

2) En cuanto al sujeto que la realiza: una tercera persona en el caso del CT (traductor interno) y de la norma LISA QA Model (Quality controler) y el propio traductor (autorrevisión) en la norma alemana DIN $2345^{2}$.

En cuanto al segundo objetivo, conocer la relación que existe entre la revisión de traducciones y otros procedimientos para valorar la calidad de la traducción, en el caso del cт, los procedimientos inherentes a su sistema de calidad son, siguiendo el orden en el que se llevan a cabo: I) El control de calidad mediante muestras seleccionadas al azar en todo el documento, 2) La lectura de verificación del texto de llegada completo y 3) La revisión.

En la norma LISA QA Model, los procedimientos de calidad que prevé la norma, en el transcurso de la ejecución del proyecto de traducción, son: I) El aseguramiento de la calidad (AC) mediante el control de muestras del texto (una primera vez, antes de proceder al control de calidad), 2) El control de calidad (cc) mediante una revisión completa del TL y 3 ) El aseguramiento de la calidad (AC) mediante el control de muestras del texto ${ }^{3}$.

Por último, dado que la finalidad de la norma

I La norma LISA QA Model, sistema para garantizar el aseguramiento de la calidad en el sector de la localización que también se utiliza para los proyectos de traducción, requiere que, durante la ejecución del proyecto de traducción, una persona que no sea el traductor realice una revisión del TL completo, mediante el denominado control de calidad (cc). De acuerdo con esta norma, el cc consiste en la verificación del TL completo, es decir, en una revisión, y no en la verificación de una o varias muestras del TL, procedimiento denominado en dicha norma como aseguramiento de la calidad (AC).

2 Salvo que se acuerde con el cliente que sea un tercero quien se ocupe de hacerla.

3 Cuando el TL adquiere la categoría de producto final, es decir, una vez realizado el control de calidad y las modificaciones correspondientes. 
DIN 2345 es completar y regular los contratos entre el cliente y el traductor para la prestación de servicios de traducción, tienen la consideración de procedimiento de calidad en dicho sistema: I) La documentación relativa al contrato de traducción (el tipo de información y datos que deben constar en la documentación del proyecto de traducción) y 2) La autorrevisión4.

Con relación al tercer objetivo, podemos concluir que la revisión de la traducción es un procedimiento más exhaustivo y fiable que el cc mediante la selección de muestras para garantizar la calidad del TL.

Los argumentos para justificar la anterior afirmación, con respecto a los casos analizados, son los siguientes: I) En el Centro de Traducción se revisan las traducciones en cuanto se detectan problemas serios de calidad y la norma LISA QA Model establece la obligatoriedad de revisar el TL, una vez superado con éxito el AC mediante el control de muestras y 2) La norma DIN 2345 estipula que la revisión del TL por una tercera persona tendrá la consideración de una prestación complementaria ${ }^{5}$.

Finalmente, como conclusión global respecto a los parámetros de revisión que utilizan los tres sistemas de calidad estudiados, estimamos que:

a) En teoría, no existen diferencias importantes en cuanto a la denominación de los parámetros de revisión que determinan los tres sistemas de calidad

b) En la práctica, dado que la denominación

4 En nuestra opinión, el hecho de que un traductor cumpla los requisitos de la norma DIN 2345, en cuanto al procedimiento de documentación, no implica necesariamente una garantía de calidad respecto al producto final (TL).

5 De hecho, esta prestación adicional se factura por separado puesto que supone un valor añadido para garantizar al cliente la calidad de la traducción.

de los parámetros es de mera referencia, hemos observado que la forma de aplicar los parámetros, con respecto a los aspectos que se han de verificar en el TL, no siempre coincide en los tres sistemas o, simplemente, no se determina.

\section{PRINCIPIOS GENERALES PARA LA REVISIÓN DE TRADUCCIONES}

Al estudiar las obras monográficas sobre la revisión, hemos constatado que todos los autores tratados (Brunette, Horguelin, Hosington y Mossop) abordan en primer lugar los parámetros de la revisión y después los principios por los que se rige. Sin embargo, nosotros procederemos a la inversa: primero estableceremos las bases de las que partimos, es decir, los principios generales de la revisión y, a continuación, determinaremos los parámetros que nos servirán de referencia para revisar la traducción.

La premisa básica para revisar una traducción es, a nuestro juicio, conocer el encargo de traducción. Seguidamente, por una cuestión meramente práctica, lo lógico es proceder a la lectura del TL como si de un texto de origen (то) se tratase. En el supuesto de que en esa primera lectura se detecten errores, a priori, un traductor con amplia experiencia puede deducir si merece la pena o no corregir la traducción, o sea, si la revisión del TL será o no rentable. En caso afirmativo, se determinará el grado de revisión que requiere el TL y qué parámetros de revisión se van a utilizar, en función de las especificaciones (explícitas e implícitas) del encargo de traducción. A continuación, se corregirán los errores detectados y se realizarán las mejoras oportunas en el TL, siempre y cuando se puedan justificar. Finalmente, el revisor asumirá la responsabilidad de indicar si el TL puede considerarse listo para su entrega al cliente.

La anterior reflexión contiene, grosso modo, 
los principios básicos de los que, en nuestra opinión, debe partir el revisor para delimitar su intervención. Seguidamente, explicamos, de forma somera, como articulamos y justificamos estos siete principios de revisión (Véase un resumen de los principios de revisión en el cuadro $\mathrm{n}^{\circ} \mathrm{I}$ ).

I. Conocer el encargo de traducción.- Es fundamental que, antes de emprender su tarea, el revisor disponga de una información lo más completa posible sobre los aspectos que rodean la producción de la traducción: finalidad, destinatario y posible difusión del TL; circunstancias relativas a la producción del ro (autor, fecha, finalidad, destinatario, tipo de texto); condiciones de entrega del TL (plazo, presentación, formato, honorarios) y tiempo disponible para la revisión ${ }^{6}$.

2. Leer el TL como si de un to se tratase.Dada la importancia de la relación coste/beneficio en el ámbito profesional, lo más práctico para economizar tiempo, una vez conocido el encargo de traducción, es leer el TL completo (o una o varias muestras) intentando asumir el papel del destinatario, dado que este último no recurre al то. Si el revisor se ve obligado a remitirse al то para comprender el mensaje, la traducción no se puede considerar funcional por no cumplir con un requisito mínimo e implícito para cualquier encargo de traducción: que la comunicación sea eficaz. Comenzar a valorar la calidad de la traducción mediante la lectura del TL permite rechazar, de entrada, los textos de ínfima calidad o muy mediocres, lo cual supone ganar tiempo.

6 Este principio viene a corresponder al de Mise en situation formulado por Horguelin (1985: 43).
3. Estudiar la rentabilidad de la revisión.En función de las veces que el revisor tenga que recurrir al To y del tipo y número de errores que se detecten en esa primera lectura del TL o de fragmentos de éste, se podrá deducir si la revisión es rentable o no. No obstante, el revisor debe ser consciente de que mediante la lectura de verificación del TL o, si es el caso, la lectura de muestras de la traducción, sólo puede identificar determinado tipo de errores (sinsentidos, incoherencias, gramaticales, ortográficos, de sintaxis y tipográficos), al no tratarse de una revisión comparativa. Si el revisor estima que la revisión no es rentable, tiene dos opciones: devolver la traducción al traductor para que la revise o, si es pésima, que otro traductor vuelva a traducir el To.

4. Determinar la modalidad de revisión y el grado de revisión que requiere el TL.- $\mathrm{Si}$ el revisor estima que la revisión del TL es rentable, a continuación, deberá decidir el grado de revisión que requiere el TL y los parámetros que va a utilizar, o sea, la modalidad de revisión más apropiada. Para ello deberá considerar, al menos, los siguientes factores: las especificaciones explícitas e implícitas del encargo de traducción, el tiempo disponible para la revisión, la experiencia y cualificación del traductor $y$, finalmente, sus propios conocimientos sobre el tema.

5. Minimizar las modificaciones en el TL.Cualquier modificación en el TL, ya se trate de correcciones o mejoras, requiere tiempo. Por este motivo, cuando el tiempo disponible es escaso, el revisor debería realizar las modificaciones estrictamente necesarias, a saber: las relativas a la corrección de los errores. Con respecto a las mejoras estilísticas, en caso de duda, el revisor debe abstenerse de cambiar el texto y prestar especial atención para no considerar que el uso de un idiolecto diferente al suyo propio, 
en este caso el del traductor, es sinónimo de error.

6. Justificar las correcciones.- El revisor debe tener como lema el poder justificar toda corrección o mejora, independientemente de que así lo solicite el cliente o el traductor, antes de realizar cualquier tipo de modificación en el $\mathrm{TL}^{7}$.

7. Responsabilidad del revisor.- En el caso de la revisión pragmática, el revisor es el responsable de decidir si la calidad del TL es suficiente para considerarlo listo para su entrega al cliente, dado que el traductor no tiene la oportunidad de tratar con el primero sobre la pertinencia de las modificaciones realizadas. Si existe una colaboración muy estrecha entre traductor y revisor, la responsabilidad de este último es más limitada ya que, si bien interviene para comprobar si se han cumplido las especificaciones del encargo de traducción, es el traductor quien debe realizar las oportunas modificaciones para que el TL sea aceptable. Cuando el cliente impone el cumplimiento de ciertos requisitos que el revisor no acepta (uso de una terminología concreta, registro, etc.), es importante que manifieste su disconformidad para quedar exento de cualquier responsabilidad al respecto.

\section{LOS PARÁMETROS DE LA REVISIÓN DE TRADUCCIONES}

En nuestra opinión, cualquier sistema para garantizar la calidad de la traducción en el ámbito profesional, además de determinar y clasificar los criterios de calidad y los parámetros de revisión, debería explicar, de una forma

7 Este principio es fundamental y debe cumplirse escrupulosamente en el caso de la revisión formativa y de la revisión didáctica.

clara, los aspectos que se han de verificar al aplicar el criterio en cuestión y la manera de hacerlo.

No obstante, basándonos en diversas constataciones de Mossop ${ }^{8}$ (2000: 40), podríamos concluir que, hasta que no dispongamos de resultados concluyentes a partir de estudios empíricos a gran escala, no hay nada que justifique establecer un orden rígido para aplicar los parámetros de la revisión.

Siguiendo parcialmente a Mossop 9 , hemos establecido un total de nueve parámetros de revisión que, posteriormente, hemos clasificado en cuatro grupos debido a que, de acuerdo con nuestra experiencia, hemos constatado que no es raro que se utilicen varios parámetros, de manera simultánea, para verificar la calidad del TL.

Por otra parte, estimamos que el uso simultáneo de varios parámetros para revisar la traducción se debe a la existencia de una cierta afinidad entre éstos. Esta afinidad entre determinados parámetros nos permitirá, además de clasificarlos por grupos, en función de los aspectos generales que se pretendan comprobar en el TL, determinar la modalidad de revisión que se va a realizar y el correspondiente perfil del revisor.

Así mismo, conviene subrayar que los criterios para valorar la calidad de un texto original o de una traducción son básicamente los mismos (salvo contadas excepciones) puesto que el

A saber, la diferencia entre las fases y actividades que implica el proceso de traducción y el hecho de que los traductores distribuyan las actividades necesarias para la producción del TL entre las fases, incluida la de autorrevisión, de diferente forma y en función de diversos factores (Mossop, 2000: 40).

9 Mossop (200Ia) establece I2 parámetros clasificados en cuatro grupos. Véase también Parra Galiano, 2005: 142I 45 . 


\begin{tabular}{|c|c|}
\hline Principio & Contenido Premisa \\
\hline 1. Conocer el encargo de traducción & $\begin{array}{l}\text { - Finalidad, destinatario, difusión del TL } \\
\text { - Aspectos situacionales relativos a la produc- } \\
\text { ción del To (autor, fecha, finalidad, destinata- } \\
\text { rio, tipo de texto) } \\
\text { - Condiciones de entrega y tiempo disponible } \\
\text { para la revisión }\end{array}$ \\
\hline 2. Leer el TL como si de un TO se tratase & $\begin{array}{l}\text {-Comenzar la revisión con la lectura del TL } \\
\text { - Asumir el papel del destinatario } \\
\text { - No recurrir al тo }\end{array}$ \\
\hline 3. Estudiar la rentabilidad de la revisión & $\begin{array}{l}\text { - Análisis somero del tipo y número de errores } \\
\text { detectados en el TL } \\
\text { —Estimación del tiempo de revisión } \\
\text { - Toma de decisión: revisar, devolver al traduc- } \\
\text { tor, volver a traducir (otro traductor) } \\
\end{array}$ \\
\hline $\begin{array}{l}\text { 4. Determinar la modalidad y grado de } \\
\text { revisión que requiere el TL }\end{array}$ & $\begin{array}{l}\text { En función de: } \\
\text { —especificaciones del encargo (explícitas e } \\
\text { implícitas) } \\
\text { —experiencia y cualificación del traductor } \\
\text { — tiempo disponible y } \\
\text { —conocimientos del revisor sobre el tema } \\
\end{array}$ \\
\hline 5. Minimizar las modificaciones en el TL & $\begin{array}{l}\text { - Corregir primero los errores evidentes } \\
\text {-En caso de duda abstenerse de modificar el TL } \\
\text { - Tener presente la: diversidad de idiolectos y la } \\
\text { diferencia entre corrección y mejora del TL }\end{array}$ \\
\hline 6. Justificar las correcciones & $\begin{array}{l}\text { - No modificar el TL si no existe un argumento } \\
\text { de peso }\end{array}$ \\
\hline 7. Responsabilidad del revisor & $\begin{array}{l}\text {-Asumirla si no existe relación con el traductor } \\
\text { - Compartirla cuando la colaboración con el } \\
\text { traductor es estrecha } \\
\text { Rechazarla en el supuesto de imposiciones del } \\
\text { cliente que el revisor no acepta }\end{array}$ \\
\hline
\end{tabular}


objetivo final en ambos casos es la transmisión eficaz de un mensaje.

Dado que la denominación que ofrecemos 204 para cada parámetro es de mera referencia, seguidamente expondremos los aspectos que, en nuestra opinión, debe comprobar el revisor o el traductor (autorrevisión) al utilizar cada uno de los parámetros que hemos establecido.

\section{I Parámetros relativos al contenido}

Como ya hemos indicado, creemos más práctico iniciar la revisión leyendo la traducción porque mediante esta lectura de verificación del TL resulta relativamente fácil apreciar si, entre otros, existen errores relativos a la lógica del texto, a los datos que contiene y a la terminología y fraseología utilizada por el traductor, sobre todo, cuando el To versa sobre un tema muy especializado. En esta situación, es recomendable que el revisor posea unos conocimientos amplios sobre el tema. De lo contrario, es muy probable que el revisor en cuestión no advierta la existencia de tales errores, por lo que es preferible que la persona que se ocupe de verificar los aspectos relativos al contenido sea un experto en la materia.

\section{I.I Lógica}

Mediante el uso de este parámetro, el revisor debe verificar si el TL está bien construido, desde el punto de vista formal o lingüístico y desde el punto de vista semántico. En consecuencia, la verificación de este parámetro depende tanto de la coherencia, como de la cohesión del TL.

Para que la traducción sea lógica, de principio a fin, debe tener pleno sentido para el lector en su correspondiente contexto. Por lo tanto, en la secuencia de ideas no deben existir contrasentidos o sinsentidos.

Las anomalías relativas a la lógica del $\mathrm{TL}$ pueden estar motivadas por dos causas: a) porque el to o una parte de éste carece de lógica y b) porque el traductor ha cometido un error al transferir el sentido del то al TL. En el primer supuesto, el traductor debe dirigirse al cliente o al autor del то para resolver el problema antes de hacer la traducción. El segundo caso es típico en traductores principiantes, normalmente debido a un conocimiento insuficiente de la LO o del tema, y en los más experimentados debido a la prisa o al cansancio.

\section{I.2 Datos}

Para identificar la existencia de posibles errores factuales, conceptuales, numéricos o matemáticos en el TL, es obvio que, si no se puede contar con un experto en la materia, el revisor debe conocer el tema. Este tipo de errores pueden encontrarse en el to o ser introducidos en el TL por el traductor.

En el primer caso, es necesario informar al cliente o consultar al autor del to antes de realizar cualquier corrección, dado que estos errores son de vital importancia para la comunicación. Si el autor del to no se aviene a razón y el error detectado es flagrante, conviene indicar, de alguna manera, que el error en el TL no se debe al traductor puesto que, de lo contrario, se cuestionaría tanto la competencia del autor del To, como la del traductor y el revisor. Al aplicar este parámetro conviene prestar una atención especial a las cifras.

\section{I.3 Lenguaje especializado}

$\mathrm{Al}$ revisar el TL en cuanto al uso del lenguaje especializado por parte del traductor, el revisor deberá considerar no sólo la terminología, sino la fraseología y la estructura del documento. Cada género y tipo de texto recurre al uso de sus propios recursos léxicos, sintácticos y retóricos en la LL que, por lo demás, no tienen por qué coincidir ${ }^{\mathrm{ro}}$ con los de la Lo. Si el revisor no 
conoce el tema con profundidad, es importante que recurra a textos paralelos, redactados en la LL, para comprobar si la terminología y fraseología del TL son las apropiadas.

\subsection{Parámetros relativos al lenguaje}

Como ya hemos comentado, algunos de los parámetros que proponemos se suelen utilizar para verificar en el TL diferentes aspectos generales. En este sentido, el parámetro del lenguaje especializado debería utilizarse tanto al comprobar los aspectos relativos al contenido, como los aspectos lingüísticos. Sin embargo, cuando el revisor no está familiarizado con el tema, por ser muy especializado, la revisión respecto al uso de la terminología y fraseología de un determinado lenguaje de especialidad debería hacerla una persona con los conocimientos apropiados. En este caso, el revisor debería limitarse a verificar los aspectos relativos al lenguaje utilizando para ello los dos parámetros siguientes.

\subsection{Norma y uso de la lengua de llegada}

Al utilizar este parámetro, el revisor debe asegurarse de que el traductor ha respetado el código lingüístico de la LL, recurriendo para ello a las nociones de norma y uso vigentes. Antes de realizar cualquier modificación en el $T L$, el revisor debe tener presente la diferencia entre corrección y mejora, así como la diversidad de idiolectos. Además de comprobar si los enunciados son claros y concisos, para que la comunicación sea eficaz, debe prestar una atención especial al estilo y comprobar que las expresiones y combinaciones terminológicas utilizadas son idiomáticas en la LL.

\footnotetext{
ro A modo de ejemplo baste con mencionar el uso de los tiempos verbales en los documentos notariales redactados en francés y español. Mientras que en el primer caso se utiliza el passé composé (pretérito perfecto compuesto) en el segundo se usa el presente.
}

Finalmente, el revisor verificará si el traductor ha utilizado el tono apropiado, es decir, un nivel de lengua y registro similares a los del то, siempre y cuando el perfil del destinatario del TL sea parecido.

\subsubsection{Adaptación al destinatario}

Si el perfil del destinatario del то no coincide con el del TL, el revisor debe comprobar si el traductor ha modificado el tono mediante un nivel de lengua y registro apropiados. Además deberá asegurarse de que el grado de fluidez (cohesión) del TL se ajusta al destinatario y a la finalidad de la traducción. En este sentido no se puede olvidar que la falta de fluidez del тo no justifica la de la traducción y que la fluidez del TL vendrá determinada por el usuario y destino de la traducción, no por el grado de fluidez del To. El revisor debe tener presente que el lector tiene que captar el sentido del texto en la primera lectura realizada a una velocidad normal. De no ser así, es posible que la conexión entre frases sea pobre, que la relación sintáctica no sea clara o que el traductor haya calcado el orden de palabras y los conectores del To.

Por otra parte, el revisor debe verificar si el traductor ha hecho lo posible por minimizar en el TL el número de palabras (frases, ejemplos y nombres) en la LO, relacionados estrechamente con alusiones y referencias a la cultura de origen (CO), ya que pueden afectar negativamente a la fluidez del TL.

\subsection{Parámetros relativos a la finalidad del texto de llegada}

Un requisito que debe cumplir cualquier traducción es que transmita de manera fiel y exacta el sentido del то. Para comprobar lo anterior, es imprescindible comparar de manera exhaustiva ambos textos y, además, considerar cuál es la función del TL y el destinatario del mismo. 
Es evidente que esta revisión comparativa sólo podrá realizarla, de manera eficaz, alguien que posea una competencia traductora. Esta macrocompetencia traductora, como la denominan algunos autores ${ }^{\text {II }}$, implica, entre otros, un profundo conocimiento de las lenguas en presencia, de la cultura de origen (CO) y de la cultura de llegada (CL), así como de las técnicas y estrategias de traducción. Para verificar si el TL cumple su función, el revisor-traductor debe aplicar el parámetro adaptación al destinatario y comprobar los dos siguientes.

\subsection{Exactitud}

Tradicionalmente, la exactitud ha sido considerada como la característica más importante de una traducción. El revisor debe garantizar que el sentido del TL es el mismo que el del тo. No obstante, de acuerdo con Mossop (200ra: Ioo) y Kussmaul (1995), la traducción no tiene que ser lo más precisa posible, sino tan precisa como requiera el uso que se hará de la misma.

El origen de la mayoría de las imprecisiones se encuentra en una comprensión incorrecta del To. Sin embargo, el TL puede contener imprecisiones incluso cuando el traductor ha comprendido correctamente el To.

En este último caso, el problema radica en una transferencia inapropiada que, en muchas ocasiones, se materializa en frases ambiguas desde el punto de vista semántico. La revisión de la traducción, frente a la autorrevisión, se muestra en este caso como el mejor instrumento para detectar esa ambigüedad tan peligrosa pero que resulta necesaria, en determinadas situaciones, debido a razones políticas e ideológicas.

Con relación a la legibilidad del TL, estamos

II Cf. Kelly (2002) y Presas (2000).

parcialmente de acuerdo con Mossop en que, en ciertos casos, cuanto mayor es el grado de precisión menor es la legibilidad y viceversa ${ }^{\mathrm{I2}}$ (200ra: IOI). La clave está en lograr el equilibrio entre la exactitud y la legibilidad, en función del encargo de traducción.

\subsubsection{Integridad}

Salvo si el encargo de traducción consiste en hacer una traducción resumen ${ }^{\mathrm{I} 3}$ o una traducción parcial del To, en principio, el TL debe transmitir la totalidad del mensaje que contiene el тo. Por este motivo, el revisor deberá asegurarse de que la traducción cumple el Principio AIOI, es decir, que el TL no ha sido objeto de adiciones innecesarias ni de omisiones innecesarias. No obstante, en ciertos casos, algunas adiciones y omisiones son inevitables. El cumplimiento del parámetro de la integridad del TL puede exigir añadir explicaciones de tipo cultural o técnico; en otros casos, el TL no requiere reproducir, por ejemplo, las repeticiones típicas de un to mal escrito, ni las explicitaciones de unidades de sentido del to que ya han aparecido implícitas en el TL.

Finalmente, siguiendo un práctico consejo de Mossop (200Ia: I03-IO4 y 200Ib) con relación a los textos entregados en soporte electrónico, es importante que el revisor verifique que también se han traducido los elementos de texto ocultos (forms of bidden writing) que no aparecen visibles de forma inmediata ${ }^{\mathrm{I}}$.

\footnotetext{
I2 "At the higher levels of precision, a degree of readability is inevitably sacrificed, while at the higher levels of readerfriendliness, accuracy must suffen (Mossop 200Ia: IoI).

I3 Este tipo de traducción suele ser muy demandada en el ámbito jurídico y económico (informes) así como por las personas que publican en revistas especializadas, ya que normalmente se exige la presentación de un resumen o abstract redactado en una lengua diferente a la del artículo en cuestión.

${ }^{14}$ Téngase en cuenta que, por ejemplo, los encabezamientos y notas a pie de página no se ven si seleccionamos
} 


\subsection{Parámetros relativos a la presentación del TL}

Como hemos visto antes, la exactitud o precisión del TL conlleva, salvo en determinados casos, la traducción íntegra del to. Debido a la facilidad con la que se puede omitir algo de forma inconsciente (por ejemplo, un párrafo o incluso una página completa), si el documento es muy extenso, es aconsejable verificar por separado el parámetro relativo a la integridad para garantizar que no existen omisiones accidentales.

Una posible forma de comprobar que se ha traducido íntegramente un documento es mediante el recuento de capítulos, apartados, epígrafes y párrafos, y cotejando el índice y la bibliografía para verificar, al mismo tiempo, si se han respetado las especificaciones relativas a la presentación física del TL. Sin embargo, esta manera de comprobar la integridad del TL es sólo relativa, ya que la transmisión íntegra del sentido del To sólo se puede comprobar a través de una revisión comparativa.

Para verificar el cumplimiento de los aspectos relativos a la presentación del TL como, por ejemplo, que no se ha omitido ningún párrafo y que la disposición textual y la ortotipografía son adecuadas, no es imprescindible que el revisor sea un traductor. En principio, cualquier persona con la experiencia y conocimientos necesarios de ortotipografía y gramática, así como de maquetación y compaginación, si se trata de un libro o de documentos muy extensos, puede ocuparse de verificar estos aspectos.

la opción «ver»y, a continuación, «normal» de Word para visualizar el texto en pantalla.

\subsection{Maquetación}

El revisor deberá verificar asimismo si la estructura del texto y su disposición global es clara y no induce a confusión, dada la importancia de los aspectos relativos a la maquetación del documento para su lectura. En este sentido, es importante asegurarse de que la numeración relativa a los cuadros, tablas, capítulos, etc., que aparece en el índice corresponde realmente a las páginas de referencia.

Por otra parte, se verificará si la compaginación o disposición de las páginas es adecuada $\mathrm{y}$, en su caso, conforme a las normas dictadas por el cliente al respecto, siempre y cuando éstas no sean contrarias al uso establecido en la LL. Para ello conviene comprobar si los márgenes, interlineado, espacio entre columnas, secciones y tablas, por ejemplo, son homogéneos. Estas comprobaciones son muy importantes cuando el To y el TL aparecen editados en un mismo documento (normalmente a doble columna) ya que el lector suele comparar ambos textos.

\subsubsection{Ortotipografia}

El revisor debe verificar si en el TL se han utilizado de manera apropiada los elementos tipográficos (cursiva, versalita, negrita, subrayado, sangría, espacio, interlineado, etc.) y, si así lo requiere el encargo de traducción, si se han respetado las normas de un determinado libro de estilo y código tipográfico ${ }^{15}$. Además, el revisor deberá corregir las erratas o letras mal colocadas, repetidas, omitidas, etc., así como las palabras mal compuestas o con cualquier tipo de error material que pueda producirse al componer el texto.

Por otra parte, es importante señalar que la misión del revisor no consiste en corregir las faltas de ortografía, ya que el traductor debe conocer perfectamente las reglas ortográficas de la LL. 


\section{MODALIDADES DE REVISIÓN}

Los parámetros que acabamos de presentar, divididos en cuatro grupos, atendiendo a los aspectos generales que, en cada caso, se hayan de verificar en el TL, considerando el encargo de traducción, dan lugar a cuatro modalidades de revisión. De ahí que el uso de una serie de parámetros de revisión, que presentan cierta afinidad, implique la práctica de diversas modalidades de revisión que hemos denominado:

I) Revisión de contenido

2) Revisión lingüística

3) Revisión funcional y

4) Revisión de la presentación del TL.

En cuanto a la finalidad de estas modalidades de revisión, hemos de señalar la existencia de un cierto paralelismo con diversas operaciones que se utilizan para perfeccionar los textos, libros y publicaciones periódicas (como la corrección de pruebas, la corrección de concepto, la corrección de estilo y la corrección tipográfica), en tanto que то. De tal forma que las intervenciones que realiza el revisor sobre un TL con respecto a aspectos de contenido, lingüísticos y relativos a la presentación (a las que hemos denominado revisión de contenido, revisión lingüística y revisión de la presentación, respectivamente) vienen a corresponder, a grandes rasgos, a las que practican los correctores de concepto, de estilo y tipográficos sobre un то.

Sin embargo, no se puede apreciar tal paralelismo en cuanto a la revisión funcional, ya que es requisito imprescindible que el revisor confronte el TL con el TO, cosa que no ocurre en la

I5 Conjunto de normas ortotipográficas cuya finalidad es unificar criterios en la representación gráfica de los elementos impresos. Se compone de las normas de ortografía necesarias para expresarse con propiedad mediante elementos tipográficos. corrección, donde la única comparación posible es entre el то y las galeradas, compaginadas y, si es el caso, terceras pruebas.

\section{PERFIL DEL REVISOR}

A priori, se puede pensar que todo buen traductor puede ser revisor pero la cuestión no es tan simple. Para ilustrar la falta de fundamento de este presupuesto Darbelnet (I980: 397) hace uso de dos nociones importantes, la de idiolecto (idiolecte) y la de pluralismo de uso (pluralisme de l'usage).

Cada persona posee su idiolecto, es decir, su forma particular de utilizar la lengua. El pluralismo de usos aceptados abarca un determinado número de variantes o idiolectos y, por este motivo, el uso específico que el revisor hace de la lengua no es el único posible, dentro de los límites del uso considerado como correcto. En algunas ocasiones, el revisor puede considerar como incorrecto un determinado idiolecto por el hecho de ser diferente al suyo.

De ahí que desempeñar la función de revisor, además de requerir un buen idiolecto, requiera un profundo conocimiento del uso correcto de la lengua y de sus variantes o idiolectos. Para que el revisor cumpla con este requisito es necesario que haya observado y reflexionado sobre el uso de la lengua y para ello, de acuerdo con Darbelnet (I980: 397), son indispensables dos condiciones: la motivación y el tiempo.

Sin embargo, creemos importante poner de manifiesto que el perfil de la persona que va a ocuparse de revisar la traducción debe depender de la modalidad de revisión que requiera el $\mathrm{TL}$, en especial, cuando no resulte posible hacer una revisión completa de la traducción. En este caso, se optará por una o varias modalidades de revisión, en función de los aspectos generales que se hayan de comprobar en el TL y que, a su vez, dependerán de las especificaciones del encargo de traducción. 
Proceder a la inversa, es decir, someter el TL a una determinada modalidad de revisión en función de la persona que en ese momento esté disponible para hacerla, sería poco profesional. Por esta razón, respecto al perfil del revisor, estimamos que lo más aconsejable sería disponer de:

I) Un revisor temático para la revisión de contenido: persona experta en el tema que aborde el TL, o que conozca bien la materia de la que trate, para verificar los aspectos relativos al contenido (lógica, datos y lenguaje especializado)

2) Un revisor lingüistico para la revisión lingüistica: persona con profundos conocimientos lingüísticos para verificar los aspectos relacionados con el uso del lenguaje en el TL (lenguaje especializado, norma y uso de la lengua y adaptación al destinatario)

3) Un revisor-traductor para la revisión funcional: un/a traductor/a con la competencia traductora y experiencia necesarias para ocuparse de verificar los aspectos relacionados con la función del TL (adaptación al destinatario, exactitud e integridad) y, finalmente,

4) Un revisor tipográfico para la revisión de la presentación: persona con la experiencia y conocimientos necesarios en tipografía, gramática y maquetación para verificar los aspectos relacionados con la presentación del TL (integridad, maquetación y ortotipografía).

\section{GRADOS DE REVISIÓN}

La revisión presenta ventajas e inconvenientes pero, indudablemente, es el procedimiento más exhaustivo y riguroso para garantizar la calidad de la traducción cuando se compara el TL completo con el To.

En el ámbito profesional, la revisión de la traducción es un servicio complementario que ofrecen las empresas de traducción porque implica un valor añadido al producto (TL).
El cliente, además de pagar los servicios del traductor, tiene que asumir un gasto suplementario, los honorarios del revisor, que no siempre está dispuesto a asumir.

Por otra parte, la revisión de la traducción conlleva un coste adicional, en términos de tiempo. No podemos perder de vista que revisar una traducción implica asimismo prever el tiempo que se necesita para hacerla y, en consecuencia, considerar este factor al determinar el plazo de entrega del TL.

Normalmente, los plazos que imponen los clientes para la entrega de la traducción suelen ser bastante ajustados. Por este motivo, los traductores y los proveedores de servicios de traducción no disponen de todo el tiempo que desean o necesitan para la prestación del servicio en las condiciones ideales y, evidentemente, este factor influye en la calidad del producto final.

Cuando los proveedores de servicios de traducción deciden que no es posible hacer una revisión completa del $\mathrm{TL}$, tienen que determinar y asumir un nivel aceptable de riesgo, con respecto a la calidad de la traducción, en función de dos factores.

El primero es de carácter económico, puesto que si el cliente no queda satisfecho es muy probable que no vuelva. El segundo es de índole profesional ya que es necesario considerar el potencial impacto que puede ocasionar cualquier error de la traducción en el destinatario de la misma. Por esta razón, cuanto mayor sea la repercusión de los posibles errores, mayor debe ser el control de calidad que se aplique a la traducción. Esto es fundamental, sobre todo, si la repercusión del error es grave o existen probabilidades importantes de que el TL presente errores de traducción u omisiones debidos, por ejemplo, a la dificultad intrínseca del texto, al desconocimiento de la competencia del traduc- 
tor o a que la traducción se haya hecho a toda prisa.

Bajo determinadas circunstancias, para 210 ahorrar tiempo y garantizar la calidad de la traducción, se puede optar por hacer una lectura de verificación del TL en lugar de una revisión completa del mismo. Sin embargo, este procedimiento implica ciertos riesgos ya que, por ejemplo, no permite detectar todas las omisiones y determinados errores de traducción. $\mathrm{El} \mathrm{TL}$ puede ser coherente y legible, a pesar de que se haya omitido la traducción de alguna frase o de un párrafo, y no transferir el sentido completo que pretendía el autor del то.

Por otra parte, cuando se opta por realizar un control de la calidad de la traducción (cc) mediante la revisión de muestras, es obvio que la parte del texto que no ha sido revisada puede contener errores.

Considerando los diferentes procedimientos que se utilizan para valorar la calidad de la traducción en el ámbito profesional, podemos concluir que el uso de algunos de estos procedimientos equivale a realizar una revisión parcial o, dicho en otras palabras, a practicar la revisión en diversos grados y modalidades.

La revisión puede ser parcial en dos sentidos:

I) Porque sólo se revisa una determinada parte o porcentaje del texto de llegada

2) Porque el revisor sólo aplica ciertos parámetros para revisar la traducción.

Entendemos por grado de revisión la variación respecto a la intensidad con la que se realiza la revisión de la traducción, en función del porcentaje o parte del texto de llegada que el revisor o el traductor analiza y compara con el texto de origen, para valorar su calidad y efectuar las oportunas correcciones y mejoras, considerando las especificaciones del encargo de traducción.
Con respecto al uso que hacemos del término «intensidad», conviene aclarar que no nos referimos a la exhaustividad o rigor con el que el revisor o el traductor utiliza los parámetros de revisión para efectuar las oportunas modificaciones en el TL.

Partiendo de la definición que hemos dado de grado de revisión, se puede establecer una diferencia entre revisión completa y revisión parcial del TL.

Dado que la revisión completa del TL implica, entre otros, comparar el roo\% de la traducción con el texto de origen, considerando el grado de revisión, nos referiremos a este procedimiento como revisión comparativa.

Por consiguiente, desde este punto de vista, el procedimiento de valoración de la calidad de la traducción que presenta un mayor grado de revisión es la revisión comparativa, en la que se analiza y compara el TL completo, o sea, el Ioo\% de la traducción, con el texto de origen.

Por otra parte, estimamos que la lectura de verificación del TL y el control de la calidad de la traducción son procedimientos en los que se realiza una revisión parcial para valorar la calidad de la traducción.

La lectura de verificación del TL, que suele realizarse en el marco del aseguramiento de la calidad (AC), presenta un grado de revisión intermedio entre la revisión comparativa y el control de la calidad de la traducción, ya que el revisor o el traductor analiza y lee el TL en su totalidad, aunque sólo recurra al To cuando detecta un problema de calidad.

El procedimiento de valoración de la calidad de la traducción que implica un menor grado de revisión es el control de la calidad de la traducción (cc), dado que el revisor o el traductor sólo analiza y compara un determinado porcentaje de muestras del TL (normalmente el ro\%) con el то. 
$\mathrm{Al}$ objeto de reflejar mejor el grado de revisión que presentan la lectura de verificación del $T L$ y el control de la calidad de la traducción (cc), proponemos utilizar en español los términos revisión unilingüe y revisión de muestras para referirnos, respectivamente, a estos dos procedimientos.

Finalmente, también se puede hablar de revisión parcial del TL en aquellos casos en los que sólo se utilizan uno o varios parámetros de revisión para verificar la calidad de la traducción y realizar las correspondientes correcciones y mejoras en el TL antes de proceder a su entrega al cliente. De ahí que, como ya hemos indicado en el apartado 5, distingamos diversas modalidades de revisión, atendiendo a los parámetros o grupos de parámetros que utiliza el revisor o el traductor.

\section{CONCLUSIÓN Y ESQUEMA DE LA PROPUESTA}

Como conclusión de lo expuesto en el presente artículo, presentamos un resumen de nuestra propuesta metodológica integrada para la revisión de traducciones y una representación esquematizada de la misma.
En la Tabla 3 figuran los nueve parámetros que hemos establecido, clasificados en cuatro grupos $^{\mathrm{T}}{ }^{6}$, atendiendo a tres criterios:

a) Los aspectos generales que debe controlar el revisor en el TL

b) La modalidad de revisión apropiada y

c) El perfil básico del revisor.

Todos los parámetros que presentamos en nuestra propuesta, concebida como una aproximación a la práctica de la revisión de traducciones en el ámbito profesional, pueden utilizarse para revisar la traducción de cualquier tipo de texto (especializado, de divulgación o literario).

No obstante, la práctica de la revisión en cualquiera de los grados mencionados (revisión comparativa, unilingüe o de muestras) y modalidades (utilizando uno, varios o todos los parámetros propuestos) dependerá de múltiples factores, entre los que destacaremos: la experiencia profesional y conocimientos temáticos del traductor, las condiciones explícitas impuestas por el cliente o las implícitas del encargo de traducción con respecto al destinatario final del TL, las económicas y las relativas al tiempo (plazo de entrega).

\begin{tabular}{|c|c|c|c|}
\hline $\begin{array}{l}\text { Procedimientos } \\
\text { DE VALORACIÓN }\end{array}$ & $\begin{array}{l}\text { PARTe del Tl } \\
\text { QUe SE REVISA }\end{array}$ & $\begin{array}{l}\text { COMPARACIÓN } \\
\text { TL/TO }\end{array}$ & Grado de ReVisión \\
\hline Revisión comparativa & completo (Iо०\%) & siempre & $\begin{array}{l}\text { R. completa / } \\
\text { grado superior }\end{array}$ \\
\hline Revisión unilingüe & completo (Io०\%) & a veces & $\begin{array}{l}\text { R. parcial / } \\
\text { grado intermedio }\end{array}$ \\
\hline Revisión de muestras & $\begin{array}{l}\text { muestra/s } \\
\text { (normalmente ıо\%) }\end{array}$ & siempre & $\begin{array}{l}\text { R. parcial / } \\
\text { grado inferior }\end{array}$ \\
\hline
\end{tabular}

Tabla 2: Grados de revisión 
La clasificación de los nueve parámetros que proponemos para revisar la traducción, en cuatro grupos, se basa por lo tanto en los aspectos generales que debe controlar el revisor en el TL. La modalidad de revisión que se realice irá en función de los mencionados aspectos que, a su vez, determinarán el perfil más apropiado de la persona que habrá de ocuparse de realizar la revisión.

A) Aspectos generales que debe controlar el revisor en el TL:

I) Relativos al contenido (parámetros: lógica, datos y lenguaje especializado)

2) Relativos al lenguaje (parámetros: lenguaje especializado, norma y uso de la lengua, $\mathrm{y}$ adaptación al destinatario)

3) Relativos a la finalidad del TL (parámetros: adaptación al destinatario, exactitud $\mathrm{e}$ integridad)

4) Relativos a la presentación del TL (parámetros: integridad, maquetación y ortotipografia)

\section{B) Modalidad de revisión}

I) Revisión de contenido (parámetros: lógica, datos y lenguaje especializado)

2) Revisión lingüistica o revisión de estilo (parámetros: lenguaje especializado, norma y uso de la lengua, y adaptación al destinatario)

3) Revisión funcional (parámetros: adaptación al destinatario, exactitud e integridad)

4) Revisión de la presentación (parámetros: integridad, maquetación y ortotipografia)

I6 En el cuadro ${ }^{\circ} 3$ puede observarse que el último parámetro de cada grupo de aspectos generales que se pueden verificar en el TL es, al mismo tiempo, el primer parámetro del grupo siguiente, considerando el orden en el que hemos representado las cuatro modalidades de revisión.

C) Perfil del revisor

I) Revisor temático para la revisión de contenido (parámetros: lógica, datos y lenguaje especializado)

2) Revisor lingüistico o revisor de estilo para la revisión lingüistica o revisión de estilo (parámetros: lenguaje especializado, norma y uso de la lengua, y adaptación al destinatario)

3) Revisor-traductor para la revisión funcional (parámetros: adaptación al destinatario, exactitud e integridad)

4) Revisor tipográfico para la revisión de la presentación (parámetros: integridad, maquetación y ortotipografía).

RECIBIDO EN MAYO 2006 Aceptado en Septiembre 2006

\section{BIBLIOGRAFÍA}

Brunette, L. (1997). Contribution à la pédagogie de la révision anglais-français en pays bilingue: le cas du Canada. Villeneuve d'Arcq: Éditions du Septentrion.

— (1998a). "La correction des traductions pédagogiques». L'enseignement de la traduction, la traduction dans l'enseignement. Delisle, Jean (Ed.), Ottawa: Les Presses de l'Université d'Ottawa (Coll.: Regards sur la traduction).

— (1998b). "L'enseignement de la révision pédagogique». Traduction et Langues de Spécialité: Approches Théoriques et Considérations Pédagogiques, $\mathrm{n}^{\circ}$ 214: 25-36.

- (2000a). "L'auto-révision - Contexte d'une formation en révision». Formation des traducteurs. Actes $d u$ colloque international Rennes 2 (24-25 Septembre 1999). En Collombat, O. y Gouadec, D., I73-I79. Paris: La Maison du Dictionnaire. (200ob). «Towards a Terminology for Translation Quality Assessment - A Comparison of TQA Practices». The Translator 6, $\mathrm{n}^{\circ}$ 2: I69-I82. (2003). Révision et mesure de la qualité des traductions. Traduction, terminologie et Rédaction. Actes des Universités d'été et d'automme(2002) et 


\begin{tabular}{|l|l|l|l|}
\hline \multicolumn{1}{|c|}{ PARÁMETRos } & $\begin{array}{c}\text { ASPECTOS GENERALES } \\
\text { QUE SE VERIFICAN EN } \\
\text { EL TL }\end{array}$ & $\begin{array}{l}\text { MODALIDAD DE } \\
\text { REVISIÓN }\end{array}$ & \multicolumn{1}{|c|}{$\begin{array}{c}\text { PERFIL DEL } \\
\text { REVISOR }\end{array}$} \\
\hline $\begin{array}{l}\text { - Lógica } \\
\text { - Datos } \\
\text { - Lenguaje especializado }\end{array}$ & De contenido & $\begin{array}{l}\text { Revisión de con- } \\
\text { tenido }\end{array}$ & Revisor temático \\
\hline $\begin{array}{l}\text { - Lenguaje especializado } \\
\text { - Norma y uso de la LL } \\
\text { - Adaptación al destinatario }\end{array}$ & Lingüísticos & $\begin{array}{l}\text { Revisión lingüís- } \\
\text { tica }\end{array}$ & Revisor lingüístico \\
\hline $\begin{array}{l}\text { - Adaptación al destinatario } \\
\text { - Exactitud } \\
\text { - Integridad }\end{array}$ & Funcionales & $\begin{array}{l}\text { Revisión funcio- } \\
\text { nal }\end{array}$ & Revisor traductor \\
\hline $\begin{array}{l}\text { - Integridad } \\
\text { - Maquetación ortotipo- } \\
\text { grafía }\end{array}$ & De la presentación del TL & $\begin{array}{l}\text { Revisión de 1a } \\
\text { presentación del } \\
\text { TL }\end{array}$ & Revisor tipográfico \\
\hline
\end{tabular}

Tabla 3: Propuesta metodológica integrada para la revisión de traducciones

du colloque international: Université de Rennes 2. Daniel y Toudic Daniel Gouadec, I23-33. París: La Maison du Dictionnaire.

Darbelnet, J. (I980). «Théorie et pratique de la traduction professionnelle: différences de point de vue et enrichissement mutuel». Meta, XXV, 4: 393-400.

Horguelin, P. A. (1985) . Pratique de la révision. $2^{\mathrm{a}}$ ed. Montreal: Linguatech.

- (I988). «La révision didactique». Meta $33, \mathrm{n}^{\circ} 2:$ 253-257.

Horguelin, P. A. y Brunette, L. (1998). Pratique de la révision, zème édition revue et augmentée. $3^{\mathrm{a}} \mathrm{ed}$. Brossard (Québec): Linguatech éditeur.

Hosington, B. M. y Horguelin, P. A. (1980). A practical guide to bilingual revision. Montreal: Linguatech.

Kelly, D. (2002). «La competencia traductora: bases para el diseño curricular». Puentes, no r: 9-20.

Kussmaul, P. (1995). Training the Translator. Amsterdam/Philadelphia: John Benjamins.

Mossop, B. (1982). «A Procedure for Self-Revision». Terminology Update, Vol I5, nº3: 6-9. Ottawa: Department of Supply and Services Canada.
- (1992). «Goals of a revision course». Teaching Translation and Interpretating. Training, Talent and Experience. Papers from the First Language International Conference. En Dollerup, C. y Loddegard, A. (Eds), 8I-90. Amsterdam/Philadelphia: John Benjamins.

- (2000). «The Workplace Procedures of professional Translators». Selected contributions from the EST Congress, Granada (1998). En Translation in Context, (eds) Chesterman, A. et al, 39-48. Vol. 39. Amsterdam/Philadelphia: John Benjamins.

- (2001a). Revising and Editing for translators. Manchester: St. Jerome.

- (200Ib). «The Translation of Hidden Quotations» [en línea]. Terminology Update, 34, $\mathrm{n}^{\circ}$ 2. Disponible en: <http://www.geocities.com/ brmossop/hiddenquotes.htm >

Parra Galiano, S. (200I). «La revisión de traducciones en la didáctica de la traducción: cara y cruz de una misma moneda». Sendebar n ${ }^{\circ}$ I2: 373-386. (2002). «Artículo bibliográfico de: Revising and Editing for Translators. Autor: Brian MOSSOP (200I). Manchester: St. Jerome Publishing. I76 pp.». TRANS. Revista de Traductología no 6: 24I247. 
(2004). «L'Évaluation de la qualité de la traduction dans le domaine professionnel: le rôle de la révision et de l'autorévision et ses implications pour la didactique de la traduction». En Georges

214 Androulaukis (Ed.), Traduire au XXème siècle: Tendances et Perspectives. Tesalónica: University Studio Press (424-43I).

- (2005). La revisión de traducciones en la Traductología: aproximación a la práctica de la revi- sión en el ámbito profesional mediante el estudio de casos y propuestas de investigación. Granada: Universidad de Granada.

Presas, M. (2000). «Bilingual Competence and Translation Competence». Developing Translation Competence. En Schäffner, C. y Adab, B., I9-31. Amsterdam: John Benjamins Publishing Co. 